\title{
Investigação sorológica de casos subclínicos de leishmaniose tegumentar após um surto em uma localidade endêmica
}

\author{
Serological investigation of subclinical cutaneous leishmaniasis \\ cases following an outbreak in an endemic area
}

\author{
Sandra Mara Alessi Aristides Arraes ${ }^{1}$, Mirela Teixeira Marini², Daiane Martello², \\ Thaís Gomes Verzignassi Silveira ${ }^{1}$, Maria Valdrinez Campana Lonardoni ${ }^{1}$ \\ e Marcos Rafael Nanni ${ }^{3}$
}

\begin{abstract}
RESUMO
Investigou-se casos subclínicos de leishmaniose tegumentar em um bairro de Maringá-Paraná, pelas reações de imunofluorescência indireta e enzimaimunoensaio. Das 130 amostras analisadas, uma amostra apresentou título 40 e três apresentaram título 20 pela imunofluorescência indireta, enquanto 11 amostras apresentaram-se positivas pela reação de enzimaimunoensaio. Estes dados sugerem a existência de casos subclínicos nesta localidade.
\end{abstract}

Palavras-chaves: Leishmaniose tegumentar. Imunofluorescência indireta. Enzimaimunoensaio. Casos subclínicos.

\begin{abstract}
Subclinical cases of cutaneous leishmaniasis were investigated in a district of Maringá, State of Paraná, by means of the indirect immunofluorescence reaction and enzyme immunoassay. Among the 130 samples analyzed, one presented titer 40 and three presented titer 20 in the indirect immunofluorescence reaction, while 11 samples were positive in the enzyme immunoassay reaction. These data suggest that subclinical cases exist at this locality.
\end{abstract}

Key-words: Cutaneous leishmaniasis. Indirect immunofluorescence. Enzyme immunoassay. Subclinical cases.

A leishmaniose tegumentar americana (LTA) é doença endêmica presente em todos os estados brasileiros ${ }^{11}$. No Estado do Paraná tem se mostrado endêmica ${ }^{3101617}$, apresentando uma incidência de 4,2\% de casos/100 mil habitantes ${ }^{12}$.

0 meio urbano da Cidade de Maringá-PR apresenta arborização exuberante com a manutenção de fragmentos florestais remanescentes de Mata Atlântica. Estes ambientes são propícios para a manutenção de fauna silvestre, atuando como possíveis focos naturais de transmissão da $\mathrm{LTA}^{19}$. No período de 2000 a 2006, foram registrados em Maringá 189 casos humanos de $\mathrm{LTA}^{13}$, dos quais 25 residiam próximos à Unidade de Conservação Parque Borba Gato ${ }^{8}$.

A transmissão da LTA em moradores próximos de unidades de conservação, indica a provável existência nesses locais de animais silvestres, reservatórios do parasita, que possibilitam a manutenção do ciclo do parasito e, conseqüentemente, a infecção do homem ${ }^{19}$.

O Laboratório de Ensino e Pesquisa em Análises Clínicas (LEPAC) da Universidade Estadual de Maringá (UEM) é Centro de Referência da Fundação Nacional de Saúde para o diagnóstico laboratorial da LTA e tem realizado os exames parasitológicos (microscopia direta) e imunológicos [intradermorreação de Montenegro (IDRM) e teste de imunofluorescência indireta (IFI)] Neste laboratório ${ }^{20}$, foi padronizado o teste de enzimaimunoensaio (ELISA) mostrando que apesar da detecção de reatividade cruzada com outras doenças, como a de Chagas, o teste foi capaz de detectar $97 \%$ de pacientes com parasitológico positivo e $97 \%$ dos pacientes com parasitológico e IDRM negativos. A reatividade

\footnotetext{
1. Departamento de Análises Clínicas, Universidade Estadual de Maringá, Maringá, PR. 2. Projeto de Iniciação Científica, Curso Farmácia, Universidade Estadual de Maringá, Maringá, PR. 3. Departamento de Agronomia, Universidade Estadual de Maringá, Maringá, PR.

Endereço para correspondência: Dr ${ }^{a}$ Sandra Mara A.A. Arraes. Av. Colombo 5790, Jardim Universitário, Zona 7, 87020-900 Maringá, PR.

Tel: 5544 3261-4878; Fax: $55443261-4860$

e-mail: smaaarraes@uem.br

Recebido para publicação em: 06/08/2007

Aceito em: 28/03/2008
} 
cruzada com a doença de Chagas foi relatada em 6,3\% dos indivíduos residentes em municípios da região $0^{6}$.

0 objetivo deste trabalho foi investigar a ocorrência de casos com sorologia positiva para LTA em familiares e vizinhos de pessoas diagnosticadas com a doença no bairro Borba Gato, município de Maringá, Paraná.

Local de estudo. Conforme mostra a Figura 1, a Cidade de Maringá (sede da 15a Regional de Saúde do Paraná) está inserida na região Noroeste do Estado do Paraná, sendo cortada pelo Trópico de Capricórnio. Possui as coordenadas geográficas: $23^{\circ} 25^{\prime}$ latitude sul; $51^{\circ} 57^{\prime}$ longitude oeste; altitude de $596 \mathrm{~m}$, com média pluviométrica anual de $1.500 \mathrm{ml}$ e umidade relativa do ar de $66 \%{ }^{14}$.

Pacientes. Foram estudados 130 indivíduos que eram da família e/ou vizinhos de pessoas que tiveram LTA, sendo todos moradores das proximidades da mata existente no bairro Borba Gato. Amostras de sangue foram coletadas para o diagnóstico laboratorial da doença e os soros armazenados a $-18^{\circ} \mathrm{C}$ até 0 uso.

Imunofluorescência-Leishmania. Foram utilizadas formas promastigotas de Leishmania (Viannia) braziliensis (MHOM/BR/1987/M11272) e conjugado anti-IgG humano-FITC ${ }^{15}$. Foram considerados como significativos títulos $\geq 40$. As amostras positivas foram testadas para anticorpos anti-Trypanosoma cruzi por imunofluorescência (IFI).
Imunofluorescência-Trypanosoma cruzi. Foram utilizadas formas epimastigotas de Trypanosoma cruzi (BioMérieux) e conjugado anti-IgG humano-FITC, considerando como significativos títulos $\geq 40$.

ELISA-Leishmania. Formas promastigotas de Leishmania (Viannia) braziliensis (MHOM/BR/1987/M11272) foram cultivadas em meio 199 (Sigma) suplementado com 10\% (v/v) de soro bovino fetal inativado, penicilina G $(100 \mathrm{UI} / \mathrm{ml})$, estreptomicina $(100 \mu \mathrm{g} / \mathrm{ml})$, L-glutamina $(0,2 \mathrm{mM})$ e $1 \%(\mathrm{v} / \mathrm{v})$ de urina humana a $25^{\circ} \mathrm{C}$. Os parasitas foram lavados com salina tamponada com fosfato (PBS) $\mathrm{pH} 7,2 \mathrm{a} 4^{\circ} \mathrm{C}$ a $1.700 \mathrm{x}$ g durante 10 minutos, liofilizados e mantidos a $4^{\circ} \mathrm{C}$. 0 preparo do antígeno e a execução do método seguiu o descrito por Yoneyama e cols ${ }^{20}$, como brevemente descrito. As placas foram sensibilizadas com o extrato diluído em tampão carbonato-bicarbonato $(0,1 \mathrm{M}, \mathrm{pH}$ 9,6). Os soros foram diluídos a 1:150 e adicionados à placa. A reação foi revelada com conjugado anti-IgG humano peroxidase e OPD (o-phenylenediamine dihydrochloride, Sigma), parada com de $\mathrm{H}_{2} \mathrm{SO}_{4} 3 \mathrm{M}$ e a absorbância medida em 492nm na leitora de microplacas Anthos 2010 (Labtec). Cada amostra foi analisada em duplicata e foram consideradas positivas aquelas com absorbância média superior a 0,68 (cutt off $)^{20}$. Em todas as placas foram incluídos controles positivos e negativos para verificação da reatividade do ensaio.

Aspectos éticos. 0 trabalho foi desenvolvido de acordo com a Resolução 196/96 do CNS/MS, submetido ao Comitê Permanente

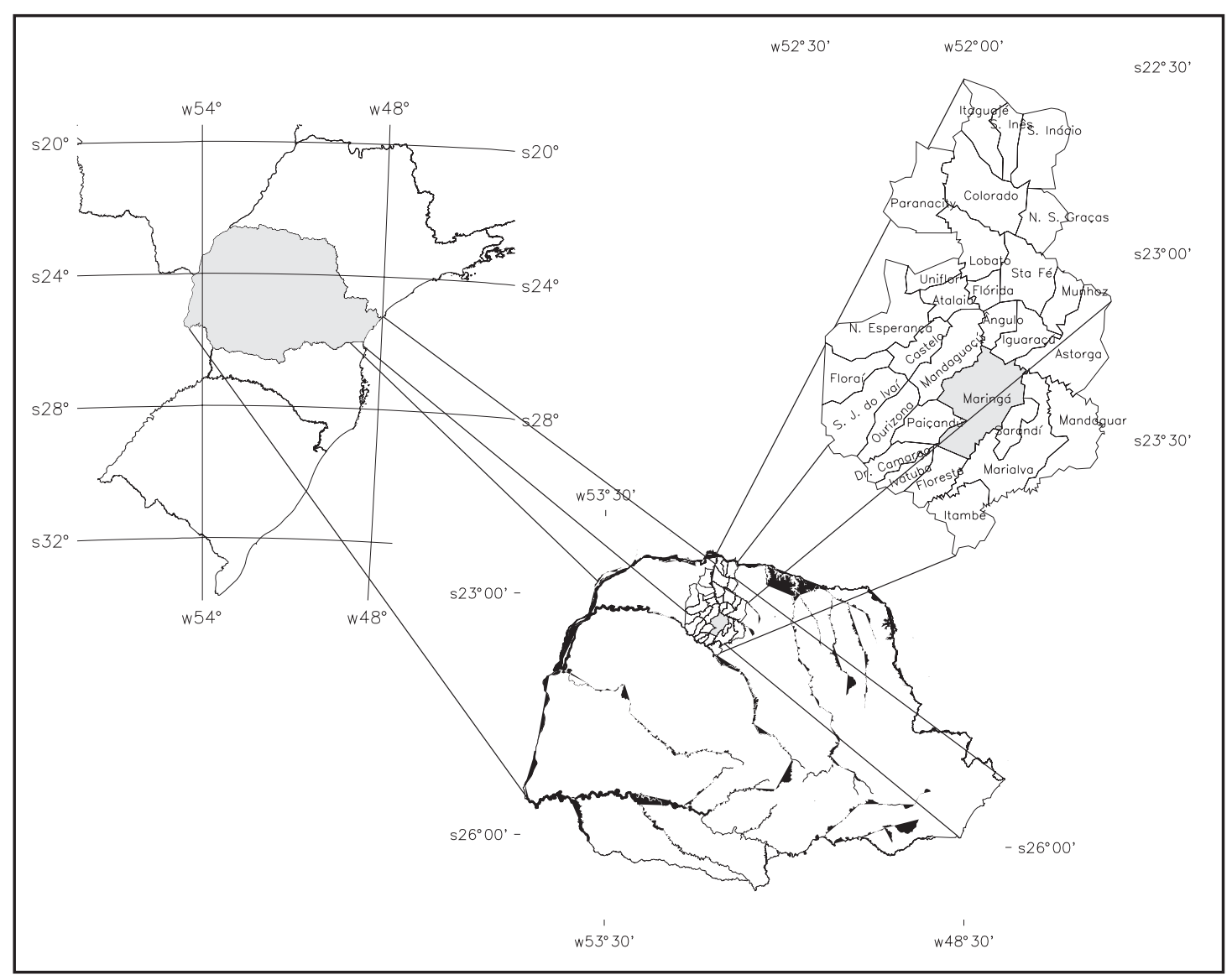

Figura 1 - Localização da Cidade de Maringá no Noroeste e da $15^{a}$ Regional de Saúde do Estado do Paraná. 
de Ética em Pesquisa Envolvendo Seres Humanos (COPEP/UEM) e aprovado conforme Parecer n $\mathrm{n}^{0}$ 164/2005.

A Figura 1 apresenta a localização da Cidade de Maringá na região Noroeste do Estado do Paraná e a $15^{\text {a }}$ Regional de Saúde.

A Tabela 1 mostra os resultados das 130 amostras de soros na imunofluorescência indireta para LTA e doença de Chagas e no enzimaimunoensaio para LTA. Dos 130 moradores do bairro Borba Gato analisados, que não apresentavam lesão, três tiveram a IFI para LTA com título 20 e um com título 40 e 11 apresentaram anticorpos anti-Leishmania na ELISA. A amostra de número 58 apresentou a maior absorbância $(2,070)$ pela ELISA, também apresentou maior título na IFI-Leishmania (40) e foi a única amostra positiva (título 80) na IFI-Chagas.

Tabela 1- Títulos de anticorpos anti-Leishmania por imunofluorescência indireta e enzimaimunoensaio e anti-Trypanosoma cruzi por imunofluorescência indireta das 130 amostras obtidas de indivíduos após surto de LTA no bairro Borba Gato, Maringá-PR.

\begin{tabular}{lrrrr}
\hline Teste/título & $\leq 20$ & 20 & 40 & 80 \\
\hline IFI-leishmania $\left(\mathrm{n}^{0}=130\right)$ & 126 & 3 & 1 & - \\
\hline Teste/absorbância & & $<0,68$ & $\geq 0,68$ & \\
\hline ELISA-leishmania $\left(\mathrm{n}^{0}=130\right)$ & - & 119 & 11 & - \\
\hline Teste/título & $\leq 20$ & 20 & 40 & 80 \\
\hline IFI-Trypanosoma cruzi $\left(\mathrm{n}^{0}=11\right)$ & 10 & - & - & 1 \\
\hline
\end{tabular}

IFI: imunofluorescência indireta, ELISA: enzimaimunoensaio.

A prevalência da LTA depende do contato dos indivíduos com 0 ambiente florestal ${ }^{5} \mathrm{e}$ a população rural e periurbana de áreas endêmicas está permanentemente exposta à picada do inseto flebótomo9 ${ }^{9}$. Seis dos 11 indivíduos com sorologia positiva, moradores do Parque Borba Gato, Maringá-PR, residiam em uma mesma rua que margeia a mata.

Os testes sorológicos são pouco utilizados para o diagnóstico da $\operatorname{LTA}^{20}$, sendo necessária a associação de diferentes métodos para aumentar a positividade do diagnóstico da doenç $\mathrm{a}^{16}$. Têm sido encontradas algumas dificuldades na utilização do teste intradérmico de Montenegro para o diagnóstico da leishmaniose, como por exemplo, o uso de diferentes espécies do parasita e de diferentes formas de preparação de antígenos além da capacidade do teste em induzir reações de hipersensibilidade tardia quando realizada pela segunda vez. A reação de IFI é a mais empregada, sendo sensível, porém com possibilidades de reações cruzadas, especialmente com a doença de Chagas, o que pode explicar o resultado obtido em um dos pacientes estudados. Em pacientes com lesões entre um a seis meses de evolução é freqüente a negatividade da $\mathrm{IFI}^{7}$. A utilização de diferentes antígenos em técnicas mais sensíveis, como a ELISA, pode fornecer diagnóstico mais específico.

A presença de anticorpos anti-Leishmania detectados pela ELISA em $11(8,5 \%)$ dos 130 moradores estudados sugere a ocorrência de casos subclínicos, após o surto de leishmaniose, ocorrido em 2003-2004 neste bairro. Este percentual é semelhante ao encontrado anteriormente em áreas endêmicas de Leishmania braziliensis $^{1418}$ e de Leishmania chagasi $^{2}$ onde $10 \%$ de pessoas saudáveis tiveram a intradermorreação de Montenegro positiva.
Os resultados mostram a presença de anticorpos antiLeishmania detectados por ELISA e sugerem a ocorrência de casos subclínicos entre os moradores do bairro Borba Gato, Maringá, após o surto de LTA.

\section{REFERÊNCIAS}

1. Badaró R, Jones T, Carvalho EM, Sampaio D, Reed SG, Barral A, Teixeira R, Johnson Jr W. New perspectives on a subclinical form of visceral leishmaniasis. Journal of Infectious Disease 154: 1003-1011, 1986.

2. Carvalho EM, Barral A, Costa JM, Bittencourt A, Marsden P. Clinical and immunopathological aspects of disseminated cutaneous leishmaniasis. Acta Tropica 56: 315-325, 1994.

3. Castro EA, Soccol VT, Membrive N, Luz E. Epidemiological and clinical study of 332 cases of cutaneous leishmaniasis in the north of Paraná State from 1993 to 1998. Revista da Sociedade Brasileira de Medicina Tropical 35: 445-452, 2002.

4. Follador I, Araújo C, Bacellar O, Araújo CB, Carvalho LP, Almeida RP, Carvalho EM. Epidemiologic and immunologic findings for the subclinical form of Leishmania braziliensis infection. Clinical Infectious Disease 43: 54-58, 2002.

5. Gomes AC, Galati EAB. Aspectos ecológicos da leishmaniose tegumentar americana. 7 - Capacidade vetorial flebotomínea em ambiente florestal primário do sistema da Serra do Mar, região do Vale do Ribeira, Estado de São Paulo, Brasil. Revista de Saúde Pública 23: 136-142, 1989.

6. Gomes ML, Bertolini DA, Silveira TGV, Lonardoni MV, Arraes SMAA. Investigação sorológica da doença de Chagas e isolamento do Trypanosoma cruzi em indivíduos de cinco municípios da região noroeste do Paraná. Revista da Sociedade Brasileira de Medicina Tropical 25: (Supl III) 94, 1992.

7. Gontijo B, Carvalho LR. Leishmaniose tegumentar americana. Revista da Sociedade Brasileira de Medicina Tropical 36: 71-80, 2003.

8. Mangabeira HN, Roberto ACBS, Zanzarini PD, Venazzi EAS, Teodoro U, Silveira TGV, Lonardoni MVC, Arraes SMAA. Surto de Leishmaniose Tegumentar no Bairro Borba Gato, Maringá - Paraná. In: Anais da VII Semana de Artes, IV Mostra do Museu Dinâmico Interdisciplinar, II Mostra Integrada de Ensino, Pesquisa e Extensão e V Simpósio da Associação Paranaense para o Desenvolvimento do Ensino da Ciência -APADEC, Maringá, 2004.

9. Marzochi MCA, Marzochi KBF. Tegumentary and Visceral Leishmaniasis in Brazil - Emerging Anthropozoonosis and Possibilities for their Control. Cadernos de Saúde Pública 10 (supl 2): 359-375, 1994.

10. Ministério da Saúde. Secretaria de Vigilância em Saúde. Disponível em: http:// dtr2001.saude.gov.br/svs/epi/situacao_doencas/transmissiveis00.htm. Acessado em 30/01/2008.

11. Ministério da Saúde, Secretaria de Vigilância em Saúde. Disponível em: http:// portal.saude.gov.br/portal/arquivos/pdf/leishmaniose_2006.pdf. Acessado em $30 / 01 / 2008$.

12. Ministério da Saúde. Secretaria de Vigilância em Saúde. Disponível em: http://tabnet.datasus.gov.br/cgi/tabcgi.exe?idb2006/d0204.def. Acessado em $31 / 01 / 2008$.

13. Ministério da Saúde. Sistema de Informação de Agravos de Notificação - SINAN Disponível em: http://dtr2004.saude.gov.br/sinanweb/novo/. Acessado em 04/02/2008.

14. Prefeitura do Município de Maringá. Demografia do Município. Disponível em: http://www.maringa.pr.gov.br/cidade/cidade.php?categoria=3. Acessado em $31 / 01 / 2008$.

15. Silveira TGV, Arraes SMAA, Pereira DSP, Lonardoni MVC, Dias MLGG, Ramos M, Bertolini DA, Fressati R, Misuta NM. Avaliação da reação de imunofluorescência indireta para leishmaniose tegumentar american em pacientes da região norte noroeste do estado do Paraná - Brasil. Revista da Universidade de Maringá 12: $177-188,1990$.

16. Silveira TGV, Teodoro U, Arraes SMAA, Bertolini DA, Lonardoni MVC, Ramos M, Sobrinho NA, Roberto ACBS, Ishikawa E, Shaw J. Observações sobre o diagnóstico laboratorial de leishmaniose cutânea e sua relação com a epidemiologia da doença no Estado do Paraná, sul do Brasil. Revista da Sociedade Brasileira de Medicina Tropical 32: 413-423, 1999. 
17. Silveira TGV, Teodoro U, Lonardoni MVC, Guilherme ALF, Toledo MJ, Ramos M, Arraes SMAA, Bertolini DA, Spinoza RP, Barbosa OC. Aspectos epidemiológicos da leishmaniose tegumentar em área endêmica do Estado do Paraná, Brasil. Cadernos de Saúde Pública, 12: 141-147, 1996.

18. Souza WJS, Sabroza PC, Santos CS, Sousa E, Henrique MF, Coutinho SG. Montenegro skin tests for american cutaneous leishmaniasis carried out on school children in Rio de Janeiro, Brazil: An indicator of transmission risk. Acta Tropica 52: 111-119, 1992.
19. Teodoro U, Alberton D, Kühl JB, Santos ES, Santos DR, Santos AR, Oliveira O, Silveira TGV, Lonardoni MVC. Ecologia de Lutzomyia (Nyssomyia) whitmani (Antunes \& Coutinho, 1939) (Diptera, Psychodidae) em área Urbana do Município de Maringá, Estado do Paraná, Brasil. Revista de Saúde Pública, São Paulo 37: 651-656, 2003.

20. Yoneyama KAG, Peder LD, Lonardoni MVC, Silveira TGV. Diagnosis of American Cutaneous Leishmaniasis by Enzyme Immunoassay in Patients from Northern Paraná State, Brazil. The Brazilian Journal of Infectious Diseases 11: 315-319, 2007. 